\title{
An Effective Attributes Mining for Larger Dataset using Neural Network
}

\author{
Ms. S. A. Patil \\ Computer Science and Engineering Department \\ Ashokrao Mane Group of Institutions, Vathar, \\ Maharashtra, India
}

\author{
Mr. P. S. Powar \\ Computer Science and Engineering Department \\ Ashokrao Mane Group of Institutions, Vathar, \\ Maharashtra, India
}

\begin{abstract}
The present world is computerized world and this is necessary to utilize computerized information, for example, video, sound, pictures and so forth in different fields for different purposes. Right now, plays imperative job in each part of business, for example, business pictures, satellite pictures, and clinical pictures, etc. Image mining is testing field which broadens conventional information mining from organized information to unstructured information such as picture information. In the attribute based access control (ABAC) model, properties are the reason for controlling access to information assets. A Convolutional neural network $(\mathrm{CNN})$ is a neural system that has at least one convolutional layers and are utilized for the most part for picture preparing, characterization, division and furthermore for other auto associated information. Steganography is the procedure of concealing mystery information inside an image or message so as to stay away from location; the mystery information is then removed at its goal. This paper covers literature survey of image mining techniques in CNN.
\end{abstract}

Keywords-Image Mining, Attribute Mining, CNN, Steganography

\section{INTRODUCTION}

The quick advancement of huge information innovation has gotten increasingly more consideration. While huge information can give comfort to individuals, for instance, by proposing things they may like, it additionally brings colossal security and protection dangers. Step by step instructions to successfully ensure the security of large information assets has become a test for enormous information advancement and its application. Traits are isolated into subject characteristics, asset properties, operational qualities, and natural characteristics. The quantity of subject Unlike information assets in customary data frameworks, enormous information assets are made out of organized, semi-organized and unstructured. The qualities in the unstructured content are changed over into dark scale pictures, and the trait mining issue is changed into the two-class/paired grouping issue. A Convolutional Neural Network $(\mathrm{CNN})$ is utilized to additionally extricate the highlights and connections of the dark scale pictures, and understand the programmed mining of asset traits.

We can consist the any type of attribute which are included in dataset there is unstructured data comes to the process it may be in form of Integer, character, string or image. We have to train that data and after that the unstructured data comes into proper

form the work is started on it.

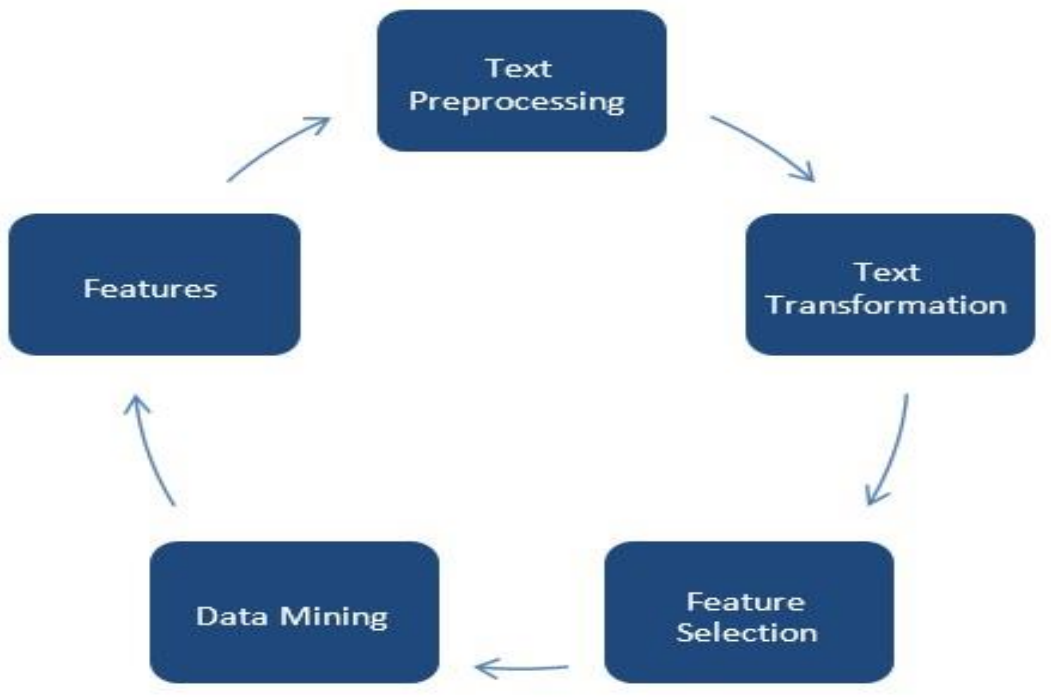

Fig: Process of Text Mining

LITERATURE REVIEW

In this paper [1] author proposed a multidimensional hybrid feature strategy for text resource properties. This technique completely figures the qualities of characteristics themselves, the connections among attributes, and the connection between 
attributes and resources. It can completely and precisely portray the attributes. It changes over characteristic highlights into grayscale pictures so as to make an interpretation of attribute mining issues into picture acknowledgement issues. This System proposes an attribute mining technique dependent on a convolutional neural system (CNN).

In the [2] proposed strategy content based retrieval utilized for recover the pictures which is generally like the input cpicture. Here taking the surface, shading and state of the picture and put away in the database. At the point when the client asks query, at that point it will be coordinated with the database and recover the picture. It will recover the specific picture by looking at the surface, shading and shape.

In this paper [3], a title-based web content extricating model TWCEM to remove the substance of each site page, which influence the title data to separate the web content. Contrasted and other extraction model, TWCEM can channel the commotions successfully and find the substance positions all the more precisely. In experiment, assess the proposed model on genuine sites, and TWCEM accomplishes best in class results and beats its rivals on both extraction execution and time cost. In propose system [4], a neural biomedical named entity acknowledgment and multi-type standardization device called BERN. (Bidirectional Encoder Representations from Transformers) named element acknowledgment models which perceive known elements and find new substances. Additionally, likelihood based choice guidelines are created to distinguish the sorts of covering substances. Besides, different named substance standardization models are incorporated into BERN for doling out a particular identifier to each perceived element. The BERN gives a Web administration to labeling substances in PubMed articles or crude content. Scientists can utilize the BERN Web administration for their content mining assignments, for example, new named element revelation, data recovery, question replying, and connection extraction.

A digital watermarking system [5] is proposed for archive copyright security and possession check with the assistance of data mining. The methods of data mining are applied to discover reasonable properties from the report for inserting watermark. The proposed model gives copyright assurance to content archives on nearby and distributed computing worldview. For the assessment of the proposed method, 20 diverse content reports are utilized to perform numerous assaults, for example, organizing, addition, and cancellation assaults.

This paper [6] estimates career versatility by consolidating text mining and item response theory (IRT), with undergrads' selfannounced profession flexibility as an abstract measure and reactions to poll things as a goal measure. The two are consolidated under a Bayesian framework. Also, the legitimacy of content classification and IRT, joined with model estimation, were investigated; content arrangement results were utilized as earlier data while evaluating IRT ability parameters to test in the case of including earlier data can improve precision.

In this paper [7] data mining approaches involves much more frameworks to separate future forecast and dynamic. In proposed system, Ensuring the capacity of picture dataset with perform hybrid strategy of information mining moved toward, for example, CBIR procedure to separate the highlights for locale of intrigue, in view of similitude of traits like shading, surface, shape, characteristic, and content area ideas and afterward manage the arrangement of pictures utilizing SVM. The presentation insights demonstrated dependent on the precision of the grouped mind pictures.

Here author [8] propose a deep neural network model consolidating convolutional neural network and provincial long shortterm memory (CNN-RLSTM) for the assignment of target-based sentiment examination. The methodology can decrease the preparation time of neural network model through a local LSTM. Simultaneously, the CNN-RLSTM utilizes a sentence-level CNN to extricate sentiment features of the entire sentence, and controls the transmission of data through various weight grids, which can viably derive the slant polarities of various focuses in a similar sentence. At last, test results on multi-area datasets of two languages from SemEval2016 and auto data show that, our methodology yields preferable presentation over SVM and a few other neural system models.

In this brief [9], the output reachable estimation and security verification issues for multilayer perceptron (MLP) neural networks are tended to. Initially, an origination called most extreme affectability is presented, and for a class of MLPs whose enactment capacities are monotonic capacities, the greatest affectability can be figured by means of taking care of convex optimization issues. At that point, utilizing a reproduction based technique, the yield reachable set estimation issue for neural networks is figured into a chain of improvement issues. At long last, a robotized security verification is created dependent on the yield reachable set estimation result. An application to the wellbeing verification for a mechanical arm model with two joints is introduced to show the adequacy of the proposed approaches.

A security attack [10] through Internet has multiplied as of late. From now on, data security is a test of intense worldwide worry of right now. The insurance of picture information from unapproved get to is basic. Encryption strategies are astoundingly important apparatuses to make sure about mystery information. Right now Effective mystery correspondence utilizing picture encryption dependent on relative change has been proposed. The mixed media encryption strategy which has incomplete encryption capacity, slight piece rate overhead, worthy security level and furthermore which is hearty to the misfortune pressure. Grasping various cryptographic calculations in current computerized correspondence structures using a variety of procedures guarantees secrecy. These interchanges were military commonly, anyway today with the development of individual correspondence structures; correspondence mystery has an incentive in military just as regular citizen correspondence situations. Computerized encryption is the primary component to guarantee the protected transmission of a message. To defeat data security issue presents a compelling mystery correspondence in forestalling cybercrimes utilizing relative change right now. 
ARCHITECTURE DIAGRAM

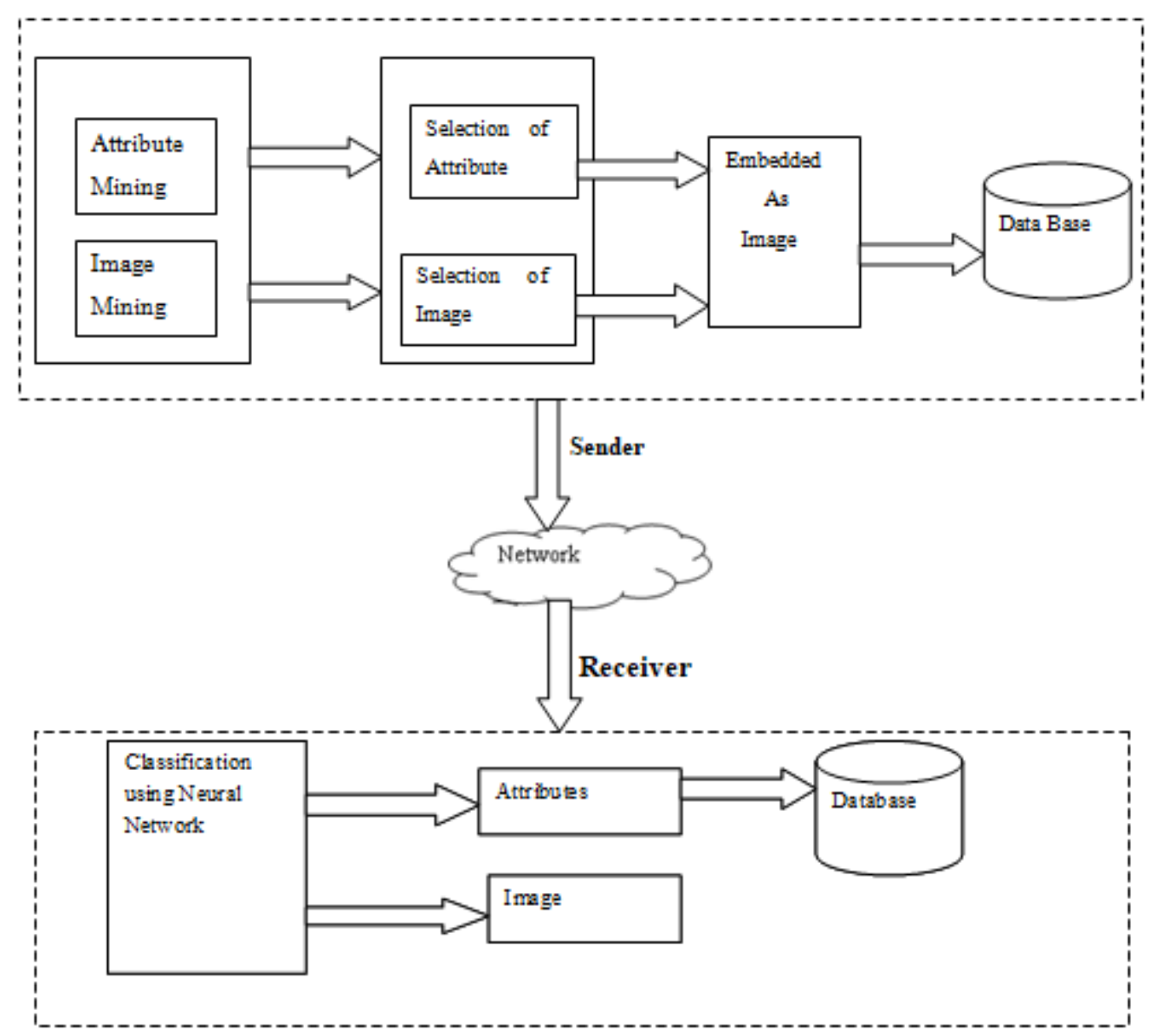

Fig: Architecture Diagram

In the proposed system Attribute mining and Image mining is considering for that will use unstructured ,structured and semi structured any type of attributes it will select as Attribute and Image using Stenography there is maintain the privacy and security and it will embed(Attributes and Images)it will saves in database this process is done in the sender side for the sending it to receiver side using the network then it will goes to the Neural Network and there it classify as Attribute and Image and Only Attribute part will save in Database.

\section{MODULE DESCRIPTION:}

\section{FIND CANDIDATE ATTRIBUTE SETS FROM TEXT RESOURCES: -}

In this module, we are going to extract attributes from text resources by apply preprocessing such as estimation of word division and part-of-speech labeling, reliance examination, named substance acknowledgment and evacuation of repetitive and invalid words, stop words and so forth. At long last creates competitor characteristic set (top 20) utilizing TF-IDF calculation. This module essentially improves precision of the trait mining technique.

In the embedding attributes and Image here is use Gray scale image attributes embedded with image for the save confidential data and provide the security for the security there is use stenography after the selection of attributes and Image the embedding process is done.

\section{EMBEDDING CANDIDATE/KEY ATTRIBUTES IN IMAGE: -}

Using LSB steganography we are going to embed set of candidate attributes in given image and store image in DB. Later on during training phase we can use these images.

\section{TRAIN USING NEURAL NETWORK: -}

In this module, we are going to train set of images (set of possible attributes) to CNN for image classification using Tensor Flow deep learning framework.

\section{ACCESS CONTROL: -}

In this module we are going to control access to text resource using given attributes, which we will classify using Neural Network and will give us access to relevant text resources. 
RESULT AND DISCUSSION:

\section{Hidden Net Neural Network Loss During Training}

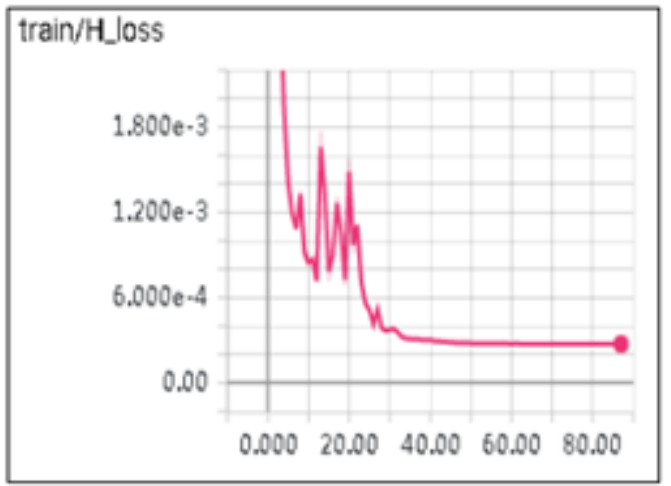

Here $Y$ axis denotes training loss where as $X$ axis denotes epoch

\section{Reveal Net Neural Network Loss During Training}

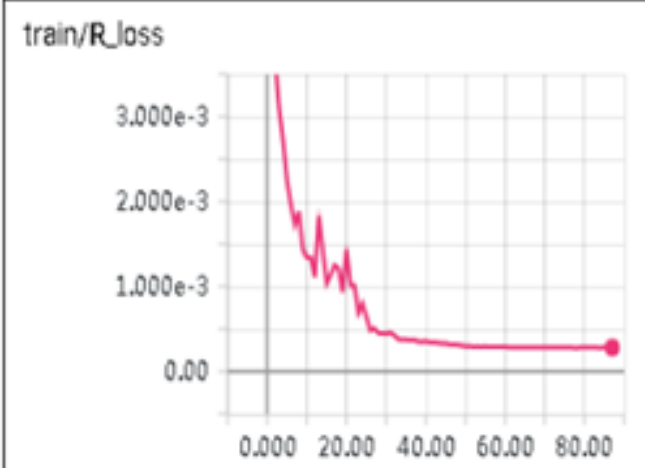

Here $Y$ axis denotes training loss where as $X$ axis denotes epoch

Fig. Train Loss

During training, as you can see the increasing no of epoch, loss gets decreased in both cases H-loss and R-loss; Minimized loss tends to accuracy of result

\section{CONCLUSION AND FUTURE SCOPE:}

In order to overcome the problems of traditional artificial attribute extraction management methods, mana em $\mathrm{nt} m$ thods, time consuming, variable accuracy and poor scalability, this paper proposes a method based on a convolutional neural network for attribute mining from big data. Its provider a new solution for The automation and intelligent mining and extraction of attributes. Such attributes might be used to support access control to unstructured big data text resources. First, we convert the candidate attributes into grayscale images. Afterwards, a CNN is used to identify and classify, he image, thereby realizing the mining of text resource attributes. The experimental results show That our CNN can automatically analyze the features of the higher dimension of thee extracted attributes And achieves better attribute mining results than existing methods but, at some cost to performance. Future scope is good quality reconstructed secret image, minimize reconstruction errors and provide less execution time

\section{REFERENCES :}

[1] AODI LIU, XUEHUI DU, and NA WANG, "Unstructured Text Resource Access Control Attribute Mining Technology Based on Convolutional Neural Network," 2019 IEEE.Translations and content mining.

[2] D.Saravanan, Dr.S. Vijaya Lakshmi, and Dr.Dennis Joseph, "Image Retrieval by image feature using Data Mining Technique," International Conference on Inventive Systems and Control (ICISC-2017)

[3] ZHEN TAN, CHUNHUI HE, YANG FANG, BIN GE, AND WEIDONG XIAO “Title-based Extraction of News Contents for Text Mining," 2019 IEEE. Translations and content mining.

[4] DONGHYEON KIM, JINHYUK LEE, CHAN HO SO, HWISANG JEON, MINBYUL JEONG, YONGHWA CHOI, WONJIN YOON, MUJEEN SUNG, AND JAEWOO KANG “A Neural Named Entity Recognition and Multi-Type Normalization Tool for Biomedical Text Mining,” 2019 IEEE. Translations and content mining.

[5] UMAIR KHADAM, MUHAMMAD MUNWAR IQBAL , MUHAMMAD AWAIS AZAM SHEHZAD KHALID, SEUNGMIN RHO, and NAVEEN CHILAMKURTI "Digital Watermarking Technique for Text Document Protection Using Data Mining Analysis," 2019 IEEE. Translations and content mining.

[6] LIHUI ZHANG, GANCHENG ZHU, SHUJIE ZHANG, XIANGPING ZHAN, WEIXUAN MENG, XIN FANG, AND PENG WANG, JUN WANG "Assessment of Career Adaptability: Combining Text Mining and Item Response Theory Method,"

[7] ReetikaKoli, Ritu Pal, Nishachaube, Kavita Joshi and AnkitMaithani “Agile Data Mining Approach for Medical Image Mining," 2019 Internationa Conference on Automation, Computational and Technology Management (ICACTM)

[8] Siyuan Chen, Chao Peng, LinsenCai and LanyingGuo“A Deep Neural Network Model for Target-based Sentiment Analysis" 2018 International Joint Conference on Neural Networks (IJCNN)

[9] Weiming Xiang, Hoang-Dung Tran and Taylor T. Johnson "Output Reachable Set Estimation and Verification for Multilayer Neural Networks" 2018 IEEE TRANSACTIONS ON NEURAL NETWORKS AND LEARNING SYSTEMS

[10] Harsh Mathur and Dr. S. Veenadhari "Blended Vector Matrix On Different Channels Of Image Encryption With Multi-Level Distinct Frequency Based Chaotic Approach To Prevent Cyber Crimes By Using Affine Transformation" 2nd International Conference on Inventive Communication and Computational Technologies (ICICCT 2018)

[11] Suraj Kumar, Santosh Kumar, Neeraj Kumar Singh, AnandaprovaMajumder and SuvamoyChangder "A Novel Approach to Hide Text Data in Colour Image".

[12] Vaibhavi S. Shukla and Jay Vala "A Survey on Image Mining, its Techniques and Application" International Journal of Computer Applications (0975 - 8887) Volume 133 - No.9, January 2016

[13] R. Suresh P. Dhivya, and N. Bhuvana "Analysis on Image Mining Techniques" 2017 International Conference on Innovations in information Embedded and Communication Systems (ICIIECS) 
[14] C. Lakshmi Devasena, T.Sumathi and Dr. M. Hemalatha “An Experiential Survey on Image Mining Tools, Techniques and Applications" International Journal on Computer Science and Engineering (IJCSE)

[15] Imran Khan, Asif Khan and Riaz Ahmed Shaikh "Object Analysis in Image Mining" 2015 2nd International Conference on Computing for Sustainable Global Development (INDIACom)

[16] BalvantTarulatha, Ms. NamrataShroff and Prof. M B Chaudhary "VIBGYOR INDEXING TECHNIQUE FOR IMAGE MINING"

[17] AbhishekVerma, Piyush Singh, and John Sahaya Rani Alex "Modified Convolutional Neural Network Architecture Analysis for Facial Emotion Recognition"

[18] Ming Zeng, Le T. Nguyen, Bo Yu, Ole J. Mengshoel, Jiang Zhu, Pang Wu, and Joy Zhang "Convolutional Neural Networks for Human Activity Recognition using Mobile Sensors"

[19] VaibhaviSolanki, Ms. Vibha Patel, and Ms. SupriyaPatil "Brain MRI Image Classification using Image Mining Algorithms" Second International Conference on Computing Methodologies and Communication (ICCMC 2018)

[20] Lipi Kothari , RikinThakkar, and SatvikKhara "Data hiding on web using combination of Steganography and Cryptography" 2017 International Conference on Computer, Communications and Electronics (Comptelix)

[21] Arnold Gabriel Benedict "Improved File Security System Using Multiple Image Steganography"

[22] Mehdi Sharifzadeh, Mohammed Aloraini, and Dan Schonfeld "Adaptive Batch Size Image Merging Steganography and Quantized Gaussian Image Steganography" 2019 IEEE Transactions on Information Forensics and Security.

[23] AnkitaTripathi, ShivamPandey and Hitesh Jangir "An improved and efficient image mining technique for classification of textual images using lowlevel image features" 